\title{
Eigenvectors and Functions of the Discrete Fourier Transform
}

\author{
BRADLEY W. DICKINSON, MEMBER, IEEE, AND KENNETH STEIGLITZ, FELLOW, IEEE
}

\begin{abstract}
A method is presented for computing an orthonormal set of eigenvectors for the discrete Fourier transform (DFT). The technique is based on a detailed analysis of the eigenstructure of a special matrix which commutes with the DFT. It is also shown how fractional powers of the DFT can be efficiently computed, and possible applications to multiplexing and transform coding are suggested.
\end{abstract}

\section{INTRODUCTION}

$\mathrm{T}$ HIS paper deals with some mathematical aspects of the discrete Fourier transform (DFT), studied with linear algebra and matrix theory methods. The vast majority of papers on the DFT have concerned computational issues, most notably the extensive literature on the fast Fourier transform (FFT) algorithms and more recently the Winograd-Fourier transform algorithm. Yet the importance of the DFT stems from more fundamental physical and mathematical principles of harmonic analysis which underlie linear signal processing technology. Thus a major goal of this paper is to present some novel ways of thinking about properties of the DFT in order to stimulate further research along more technique-oriented lines.

To be more concrete, let $F$ denote the DFT, regarded as a unitary mapping of complex $N$-space onto itself. With respect to the standard basis of $N$-space, $F$ is simply an $N \times N$ matrix whose elements are normalized complex exponentials

$$
(F)_{i k}=\left(e^{-j 2 \pi i k / N}\right) / \sqrt{N}, \quad 0 \leqslant i, k \leqslant N-1 .
$$

Thus the methods of linear algebra and matrix theory may be applied to study the DFT.

McClellan and Parks [1] suggested that the eigenstructure of $F$, being a natural linear algebraic way of decomposing the matrix, might be of computational interest as well. It was hoped that the diagonal form of $F$ with respect to an eigenvector basis of $N$-space might be exploited. Through the use of highly original methods in [1], the multiplicities of the eigenvalues of $F$ were determined and a set of eigenvectors was constructed. No computational benefits over the FFT were observed, however, and the eigenvectors obtained are not orthogonal.

It turns out that the eigenvalue multiplicity problem for $F$ has a long history, being equivalent to a problem solved by

Manuscript received August 4, 1980; revised July 29, 1981. This work was supported in part by the National Science Foundation under Grants ENG77-28523 and ENG 79-16292, and also by the U.S. Army Office of Research under Grant DAAG29-79-C-0024.

The authors are with the Department of Electrical Engineering and Computer Science, Princeton University, Princeton, NJ 08544.
Gauss [2]. This was pointed out by Good [3] and, later, McClellan [4]. Recently, Auslander and Tolimieri [5] have elaborated on the relationship of the multiplicity problem to certain constructions of abstract harmonic analysis, even showing how the FFT arises from abstract principles!

The structure of the eigenspaces of $F$ is less well understood. It appears difficult to obtain an analytical form for an orthogonal set of eigenvectors. This suggests that a computational approach may be necessary, and Yarlagadda [6] proposed one such method. In Section II of this paper, we propose a different approach based on the theory of commuting matrices. Some interesting analytical results are obtained along the way.

Functions of the DFT are discussed in Section III. The imbedding of the DFT into a family of transforms has been used by Jain [7] to study the variation of general properties of the transform family, especially for certain data compression purposes. We suggest another imbedding based on the idea of functions of a matrix. We point out that functions of the DFT matrix $F$ are simple to compute and to use in computation. The family of (fractional) powers $F^{t}$ is described in detail. Finally, a novel matrix transform pair relationship, which generalizes the DFT relationship between vectors, is constructed from fractional powers of $F$, and some possible applications are mentioned.

\section{A Method for Obtaining Orthogonal EIGENVECTORS OF THE DFT}

\section{A. The General Approach}

First, we recall some facts about the DFT matrix $F$ in (1). The minimal polynomial of $F$ is $\lambda^{4}-1$ [1], [3]-[5]; thus $F$ obeys the equation

$$
F^{4}=I
$$

where $I$ is the $(N \times N)$ identity matrix. The roots of $\lambda^{4}-I$ are distinct, so $F$ is simple or diagonalizable [8] with eigenvalues

$$
\lambda_{k}=e^{j k \pi / 2} \quad 0 \leqslant k \leqslant 3
$$

the fourth roots of unity. The multiplicities of the eigenvalues are known, as discussed in Section $I$, and are given in Table I. Since they are not distinct for $N \geqslant 4$, there are many possible sets of eigenvectors.

As motivation for our approach, suppose that we have a matrix $S$, with distinct eigenvalues, which commutes with $F$, that is,

$$
F S=S F \text {. }
$$


TABLE I

\begin{tabular}{ccccc}
\multicolumn{5}{c}{ MULTIPLICITY OF EIGENYALUES OF $F$} \\
\hline \hline $\mathrm{N}$ & $\lambda=1$ & $\lambda=\mathrm{j}$ & $\lambda=-1$ & $\lambda=-\mathrm{j}$ \\
$4 \mathrm{~m}$ & $\mathrm{~m}+1$ & $\mathrm{~m}$ & $\mathrm{~m}$ & $\mathrm{~m}-1$ \\
$4 \mathrm{~m}+1$ & $\mathrm{~m}+1$ & $\mathrm{~m}$ & $\mathrm{~m}$ & $\mathrm{~m}$ \\
$4 \mathrm{~m}+2$ & $\mathrm{~m}+1$ & $\mathrm{~m}$ & $\mathrm{~m}+1$ & $\mathrm{~m}$ \\
$4 \mathrm{~m}+3$ & $\mathrm{~m}+1$ & $\mathrm{~m}+1$ & $\mathrm{~m}+1$ & $\mathrm{~m}$ \\
\hline
\end{tabular}

Let $e$ be an eigenvector of $S$ corresponding to eigenvalue $\lambda$. Then

$$
S(F e)=F(S e)=F(\lambda e)=\lambda F e .
$$

So $F e$ is also an eigenvector of $S$ corresponding to the same eigenvalue. However, $S$ has distinct eigenvalues and, therefore, unique (up to normalization by a constant) eigenvectors [8]. Thus, for some constant $\beta$

$$
\mathrm{Fe}=\beta e
$$

so $e$ is an eigenvector of $F$ also.

If $S$ is real and symmetric, its eigenvectors will be real and orthogonal and will be the desired set of eigenvectors of $F$. The computation of the eigenvectors of a real symmetric

$$
S=\Gamma_{D}+\Delta_{D}=\left[\begin{array}{cc}
2 & 1 \\
1 & 2 \cos \omega \\
0 & 1 \\
\cdot & \\
: & \\
0 & \\
1 & 0
\end{array}\right.
$$

matrix has been thoroughly studied, and reliable methods are available [9].

From knowledge that $F$ has a set of real eigenvectors [1], we are assured of the existence of such matrices $S$. For every dimension, it would be desirable to have a canonical choice of $S$ whose eigenvectors could be obtained analytically. No solution is known for this problem. However, we will give a simple choice of $S$ whose structure is sufficiently rich to allow a complete analysis of the associated eigenproblem.

\section{B. A Class of Matrices Commuting with $F$}

Our choice for the matrix $S$ will come from a class of matrices which all commute with $F$ constructed as follows.

Let $D(z)$ be any real even FIR digital filter written in the form

$$
D(z)=a_{0}+a_{1}\left(z+z^{-1}\right)+\cdots+a_{p}\left(z^{p}+z^{-p}\right), \quad p<N / 2
$$

where time shifts are taken in the circular or $\bmod N$ sense. The action of this filter on complex $N$-space (time signals) corresponds to circular convolution or, equivalently, to multiplication by the circulant matrix $(N \times N)$

$$
\Gamma_{D}=\left[\begin{array}{ccc}
a_{0} a_{1} a_{2} \cdots a_{p} & 0 \cdots 0 & a_{p} \cdots a_{4} a_{3} a_{2} a_{1} \\
a_{1} a_{0} a_{1} \cdots & & \cdots a_{5} a_{4} a_{3} a_{2} \\
a_{2} a_{1} a_{0} \cdots & & \cdots a_{6} a_{5} a_{4} a_{3} \\
& \ddots &
\end{array}\right]
$$

Circular convolution of time signals corresponds to pointwise multiplication in the transform domain. The action of the filter $D(z)$ is thus represented by multiplication of complex $N$-vectors (transforms) by the diagonal matrix $\Delta_{D}$, which has elements

$$
\left(\Delta_{D}\right)_{k k}=D\left(e^{j 2 \pi k / N}\right), \quad 0 \leqslant k \leqslant N-1 .
$$

The correspondence between circular convolution and pointwise multiplication gives the matrix equation

$$
F \Gamma_{D}=\Delta_{D} F \text {. }
$$

From (10), using the symmetry of each matrix with respect to transposition, it follows that the matrix

$$
\Sigma_{D}=\Gamma_{D}+\Delta_{D}
$$

commutes with $F$.

This leaves the problem of determining a set of filter coefficients which leads to distinct eigenvalues for $\Sigma_{D}$. In Appendix I, we show, using perturbation methods, that such a choice is always possible. We will bypass the existence question by a careful analysis of the simplest choice of filter, $D(z)=z+z^{-1}$, corresponding to the matrix

$\left.\begin{array}{cccc}0 & \cdots & 0 & 1 \\ 1 & & 0 \\ \cos 2 \omega & & \\ & & & 0 \\ & & & 1 \\ \cdots & 0 & 1 & 2 \cos (N-1) \omega\end{array}\right]$

where $\omega=2 \pi / N$. While we have been unable to make a complete analysis, we will show that there is no difficulty in finding a complete set of real orthogonal eigenvectors for $S$ which are also eigenvectors of $F$, even when $S$ has multiple eigenvalues. Based on extensive numerical evidence, as well as the analytical results to be presented next, we make the following conjecture: the eigenvalues of the $N \times N$ matrix $S$ [(12)] are distinct except when $N$ is divisible by 4 . (When $N$ is divisible by four it can be proved that $S$ has two zero eigenvalues. We also conjecture that this is the only multiplicity which ever occurs.)

\section{The Eigenstructure of $S$}

For every $N>2$, the matrix $S$ has all of its eigenvalues in the interval $[-4,4]$ by Gershgorin's criterion [8]. To get more refined results, we will recall some facts about the relations between the eigenvalues of a matrix and those of its principal submatrices; see [9] and [10], for example.

Let $T_{i}$ be the following $i$ by $i$ principal submatrix of $S$ :

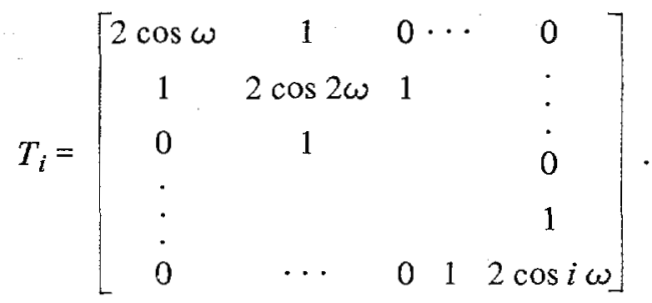


$T_{i}$ is a symmetric, tridiagonal Jacobi matrix and has distinct real eigenvalues $\left[9\right.$, p. 300]. Let $\mu_{1}>\mu_{2}>\cdots>\mu_{N-1}$ be the eigenvalues of $T_{N-1}$ and let $\lambda_{1} \geqslant \lambda_{2} \geqslant \cdots \geqslant \lambda_{N}$ be the eigenvalues of $S$. The interlacing inequalities for these eigenvalues are the inequalities $[9, \mathrm{p}, 103],[11]$, and [12]

$$
\lambda_{1} \geqslant \mu_{1} \geqslant \lambda_{2} \geqslant \mu_{2} \geqslant \cdots \geqslant \mu_{N-1} \geqslant \lambda_{N} .
$$

From the distinctness of the $\left\{\mu_{i}\right\}$ two important conclusions may be drawn: 1) no eigenvalue of $S$ has multiplicity greater than 2, and 2) any multiple eigenvalue of $S$ is also an eigenvalue of $T_{N-1}$.

The eigenvalues of $T_{i}$ are the roots of the polynomial

$$
r_{i}(\mu)=\operatorname{det}\left(T_{i}-\mu I\right), \quad i \geqslant 1 \text {. }
$$

By expanding the determinant these polynomials are found to obey the recursions $[9$, p. 423]

$$
r_{i}(\mu)=(2 \cos i \omega-\mu) r_{i-1}(\mu)-r_{i-2}(\mu)
$$

where $r_{0}(\mu)=1$ and $r_{1}(\mu)=2 \cos \omega-\mu$. A set of unnormalized eigenvectors for $T_{N-1}$ is obtained when these polynomials are evaluated at the eigenvalues of $T_{N-1}[9$, p. 316]

$$
\begin{aligned}
\left\{\left(1,-r_{1}\left(\mu_{i}\right), r_{2}\left(\mu_{i}\right), \cdots,(-1)^{N-2} r_{N-2}\left(\mu_{i}\right)\right)^{\prime}\right. & ; \\
1 & \leqslant i \leqslant N-1\}
\end{aligned}
$$

where prime $\left({ }^{\prime}\right)$ denotes transposition

Since the roots of $r_{N-2}(\mu)$ are eigenvalues of $T_{N-2}$, a principal submatrix of $T_{N-1}$, whose eigenvalues are $\left\{\mu_{i}\right\}$, the interlacing inequalities may again be applied. By considering the signs of the highest power of $\mu$ in $r_{N-2}(\mu)$, we find the values of $r_{N-2}(\mu)$ at $\left\{\mu_{i}\right\}$ satisfy

$$
\begin{aligned}
& r_{N-2}\left(\mu_{1}\right)>0, r_{N-2}\left(\mu_{2}\right)<0, \cdots, r_{N-2}\left(\mu_{N-1}\right)>0, \\
& N \text { even } \\
& r_{N-2}\left(\mu_{1}\right)<0, r_{N-2}\left(\mu_{2}\right)>0, \cdots, r_{N-2}\left(\mu_{N-1}\right)>0, \\
& N \text { odd. }
\end{aligned}
$$

Actually, more can be said. By the even symmetry of the cosine function, $T_{N-1}$ is a centrosymmetric matrix, meaning

$$
T_{N-1}=J T_{N-1} J
$$

where $J$ is the $N-1 \times N-1$ matrix with ones on the antidiagonal. By centrosymmetry, if $e$ is an eigenvector of $T_{N-1}$ corresponding to eigenvalue $\mu_{i}$, then the reversed vector $J e$ is also an eigenvector corresponding to $\mu_{i}$. Je and $e$ have the same length, and since the eigenvector is unique up to a scale factor, the form of the eigenvectors in (17) implies

$$
r_{N-2}\left(\mu_{i}\right)= \pm 1
$$

with the choice of sign determined by (18).

We are concerned with repeated eigenvalues of $S$, if any, because these may cause difficulty in determining eigenvectors of $F$. Because a repeated eigenvalue must also be an eigenvalue of $T_{N-1}$, by interlacing, we need a criterion for "inheritance of eigenvalues" of $T_{N-1}$ by $S$. Writing $S$ in partitioned form

$$
S=\left[\begin{array}{ll}
2 & a^{\prime} \\
a & T_{N-1}
\end{array}\right]
$$

where $a^{\prime}=(10 \cdots 01)$, we examine the equations required to hold if $\mu_{i}$ is to be an eigenvalue of $S$ to obtain the condition $[10$, p. 90$]$

$$
\operatorname{rank}\left[a, \mu_{i} I-T_{N-1}\right]=N-2 \text {. }
$$

There are a number of equivalent ways to express this condition [11], but because $T_{N-1}$ has eigenvectors of known form (17), the most useful is that the vector $a=\left(\begin{array}{llll}1 & 0 & \cdots & 0\end{array}\right)^{\prime}$ be orthogonal to the eigenvector of $T_{N-1}$ corresponding to eigenvalue $\mu_{i}$. (These conditions are related to controllability conditions for a particular linear system [12].) From (17) this condition becomes

$$
(-1)^{N-2} r_{N-2}\left(\mu_{i}\right)=-1 \text {. }
$$

Thus the eigenvalues inherited are the ones corresponding to eigenvectors, $e\left(\mu_{i}\right)$, of $T_{N-1}$ satisfying

$$
e\left(\mu_{i}\right)=-J e\left(\mu_{i}\right) \text {. }
$$

From (18) and (20) the number of inherited eigenvalues, and hence potential double eigenvalues of $S$ is

$$
\begin{array}{ll}
(N-1) / 2, & N \text { odd } \\
(N-2) / 2, & N \text { even. }
\end{array}
$$

We now use the condition for eigenvalue inheritance, together with the form of the eigenvectors of $T_{N-1}$, to obtain a description of the eigenspace of $S$ corresponding to an inherited eigenvalue. We show that if the multiplicity of an inherited eigenvalue of $S$ is two, then we can easily find two orthogonal eigenvectors which are also eigenvectors of $F$. This will follow from certain even/odd symmetries of the eigenvectors of $S$.

Let $\mu_{i}$ be an inherited eigenvalue of $S$. One eigenvector is then given by $u\left(\mu_{i}\right)=\left(u_{0}, u_{1}, \cdots, u_{N-1}\right)^{\prime}$ where

$$
u\left(\mu_{i}\right)=\left(0,1,-r_{1}\left(\mu_{i}\right), \cdots,(-1)^{N-2} r_{N-2}\left(\mu_{i}\right)\right)^{\prime} .
$$

This follows from the partitioned form of $S[(21)]$ and the inheritance condition [(23)]. From (24) it follows that this eigenvector has odd symmetry, in the usual (circular) sense

$$
u_{i}=-u_{-i \bmod N} \quad 0 \leqslant i \leqslant N-1 .
$$

From (21) and the distinctness of eigenvalues of $T_{N-1}$, the eigenvector $u\left(\mu_{i}\right)[(26)]$ is the only possible eigenvector of $S$ corresponding to eigenvalue $\mu_{i}$ and having a zero first component. Thus, if $\mu_{i}$ is a double eigenvalue of $S$, we may assume a second eigenvector of the form $\hat{u}\left(\mu_{i}\right)=\left(\hat{u}_{0}, \hat{u}_{1}, \cdots, \hat{u}_{N-1}\right)^{\prime}$, with $\hat{u}_{0}=1$. Again, using the partitioned form of $S[(21)]$ we obtain two equations satisfied by the eigenvector $\hat{u}\left(\mu_{i}\right)$

$$
\begin{aligned}
& \left(\mu_{i} I-T_{N-1}\right) v=a \\
& \mu_{i}-2-\hat{u}_{1}-\hat{u}_{N-1}=0
\end{aligned}
$$

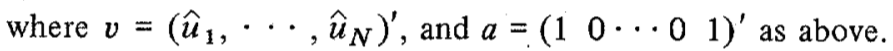
Since $a=J a$, and since the eigenvector $e\left(\mu_{i}\right)$ of $T_{N-1}$ [(24)] spans the null space of $\left(\mu_{i} I-T_{N-1}\right)$, there is a unique solution $v$ to (28a) satisfying $v=J v$. (For any solution $\widetilde{v}$, take $v=$ $(\tilde{v}+J \tilde{v}) / 2$.) If, in addition, $(28 b)$ is satisfied, then $\mu_{i}$ is a double eigenvalue of $S$. Using the reversal symmetric solution $v$ to (28a), we obtain an eigenvector $\hat{u}\left(\mu_{i}\right)$ for $S$ having even symmetry, in the sense

$$
\hat{u}_{i}=\hat{u}_{-i \bmod N}, \quad 0 \leqslant i \leqslant N-1 .
$$


The DFT is known to map even (respectively, odd) signals into even (respectively, odd) transform sequences. Therefore, by considering even and odd symmetry we are still able to conclude, as in (4)-(6), that $u$ and $\hat{u}$ are eigenvectors of $F$, even though they come from a double eigenvalue of $S$. Furthermore, $u$ and $\hat{u}$ are orthogonal since they are of opposite symmetry types.

This completes the theoretical derivation of our main result. However, there are a few other properties to be pointed out.

Using DFT real/imaginary symmetry properties, we may conclude that the even eigenvectors correspond to the eigenvalues \pm 1 of $F$ and the odd eigenvectors correspond to the eigenvalues $\pm j$ of $F$. All of the odd eigenvectors are accounted for by eigenvalues of $S$ inherited from $T_{N-1}$. Note that none of the even eigenvectors can have zero as its first component; such a vector would satisfy the inheritance condition (22), which is impossible. As a check, we can see that the number of $\pm j$ eigenvalues of $F$ from Table I equals the number of inherited eigenvalues (25).

\section{Computational Considerations}

We want to emphasize that the construction of the oddsymmetric eigenvectors of $S$, given by (26), should not be used as the basis for a computational procedure. The evaluation of the recurrence relations $[(16)]$ is highly susceptible to numerical instability [9, p. 316]. However, there are many standard numerical methods available which are well suited to obtaining the eigenvectors of $S$. For example, the eigenproblem for a class of matrices (Hill's matrices [13]) arising from the discretization of periodic boundary value problems is discussed by Bjork and Golub [14]. While this class contains matrix $S[(12)]$, there are additional symmetries which may be exploited in the eigenanalysis of $S$ arising from the centrosymmetric nature of $T_{N-1}[(19)]$.

For simplicity, we treat the case of $N$ odd, since the general case is notationally more cumbersome [15], [16]. The matrix $T_{N-1}$ can be written in partitioned form as

$$
T_{N-1}=\left[\begin{array}{ll}
A & B J \\
J B & J A J
\end{array}\right]
$$

where

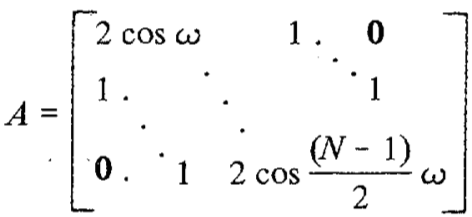

$$
\begin{aligned}
& B=\left[\begin{array}{ll}
1 & \\
& 0
\end{array}\right]
\end{aligned}
$$

and $J$ is the appropriate-sized interchange matrix as before. As above, centrosymmetry gives the eigenvectors of $T_{N-1}$ the forms $\left[x^{\prime},-x^{\prime} J\right]^{\prime}$ and $\left[y^{\prime}, y^{\prime} J\right]^{\prime}$ for vectors $x$ and $y$ of size $(N-1) / 2$. It is an easy verification that the vector $\left[x^{\prime},-x^{\prime} J\right]^{\prime}$ (respectively, $\left[y^{\prime}, y^{\prime} J\right]^{\prime}$ ) is an eigenvector of $T_{N-1}$ with eigenvalue $\mu_{i}$, if and only if the vector $x$ (respectively, $y$ ) is an eigenvector of the matrix $A-B$ (respectively, $A+B$ ) corresponding to eigenvalue $\mu_{i}[15],[16]$.
Thus, nearly half of the eigenvalues and eigenvectors of $S$ can be obtained from those of $A-B$; these are the odd eigenvectors of $S$. The rest of the eigenvalues must be found by using methods such as those described in [14]. However, the eigenvectors may be determined from half-size problems as follows. Let $v\left(\lambda_{i}\right)=\left(v_{0}, v_{1}, \cdots, v_{N-1}\right)^{\prime}$ be an eigenvector of $S$ with (noninherited) eigenvalue $\lambda_{i}$. By multiplication by $\left(\lambda_{i} I-S\right)$, we obtain

$$
\begin{aligned}
& \left(\lambda_{i}-2\right) v_{0}+v_{1}+v_{N-1}=0 \\
& \left(\lambda_{i} I-T_{N-1}\right) \bar{v}=a
\end{aligned}
$$

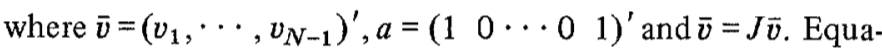
tion $(33 b)$ is easily reduced to half size by reversal symmetry.

\section{E. A Comment on Limiting Behavior}

In this section, we will show that the problem of determining the eigenstructure of the matrix $S$ is a discrete counterpart of the eigenproblem for the Mathieu equation [17], a wellknown periodic second-order self-adjoint differential equation.

Let $e=\left(e_{0}, e_{1}, \cdots, e_{N-1}\right)^{\prime}$ be an eigenvector of the matrix $S$ corresponding to eigenvalue $\lambda$. Using the form of $S$, the defining equations $(S-\lambda I) e=0$ may be rewritten in the form of a linear difference equation for the coordinate sequence

$$
(2 \cos k \omega-\lambda) e_{k}+e_{k+1}+e_{k-1}=0 ; \quad \omega=2 \pi / N .
$$

This equation will hold for all integers $k$ if we define the extended $e_{k}$ sequence to be periodic: $e_{k}=e_{k \bmod N}$. Using the central second difference operator $\delta^{2}$, where

$$
\delta^{2} e_{k}=e_{k+1}-2 e_{k}+e_{k-1}
$$

(34) becomes

$$
\delta^{2} e_{k}+(2 \cos k \omega-(\lambda-2)) e_{k}=0 .
$$

Now suppose we view $e_{k}$ as the sampled value of a continuous time signal $e(t)$ at time $t_{k}=k / N$; as $N$ gets large, (36) is the finite-difference approximation to the following second. order differential equation for $e(t)$

$$
\frac{d^{2} e(t)}{d t^{2}}+(2 \cos 2 \pi t-(\lambda-2)) e(t)=0
$$

whose periodic solutions are the Mathieu functions [17]. Thus, the eigenvectors of $S$, and, hence, the eigenvectors of the DFT, may be thought of as discrete Mathieu functions.

\section{FunCtIONS OF THE DFT AND Fractional TRANSFORMS}

We are accustomed to the usual interpretations of complex $N$-space as the time domain and of the image of this space under the DFT as the frequency domain. By using the definition of functions of a matrix, we may define the fractional DFT $F^{t}$ for $0 \leqslant t \leqslant 1$, and regard the image of $F^{t}$ as a generalization of the time and frequency domains. This will allow the consideration of generalizations of such ideas as multiplexing and transform coding.

The definition of the matrix function $F^{t}$ follows the standard approach [8] since $F$ is diagonalizable. For any function $g(F)$ we have 


$$
g(F)=\sum_{k=0}^{3} \phi_{k}(F) g\left(\lambda_{k}\right)
$$

where

$$
\lambda_{k}=e^{j k \pi / 2}, \quad 0 \leqslant k \leqslant 3
$$

and $\phi_{k}(u)$ is the Lagrange interpolation polynomial of degree 3 taking value 1 at $u=\lambda_{k}$ and value 0 at $u=\lambda_{i}, i \neq k, 0 \leqslant i \leqslant 3$. It follows that

$$
\phi_{k}(u)=\frac{1}{4} \sum_{i=0}^{3} u^{i} e^{-j k i \pi / 2}, \quad 0 \leqslant k \leqslant 3 .
$$

For the $t$ th power function, we take principal $t$ th roots of the eigenvalues $\lambda_{k}(39)$ giving

$$
\begin{aligned}
g(1) & =e^{j 2 \pi t} \\
g\left(e^{j k \pi / 2}\right) & =e^{j k \pi t / 2} .
\end{aligned}
$$

The formula for the fractional transform is

$$
F^{t}=\sum_{i=0}^{3} F^{i} \alpha_{i}(t)
$$

where

$$
\alpha_{i}(t)=\frac{1}{4} \sum_{k=1}^{4} e^{j k(t-i) \pi / 2}, \quad 0 \leqslant i \leqslant 3 .
$$

Of course, the eigenvector-eigenvalue decomposition of $F$ can also be used to define functions $g(F)$, but because $F$ has only four distinct eigenvalues and is diagonalizable, much simpler and more explicit results are obtained using (38). From (42) we see that matrix multiplication by $F^{t}$ can be accomplished efficiently by employing the FFT algorithm once (to compute the products involving $F$ and $F^{3}=F \cdot F^{2}$ ) and using linearity; multiplication by $F^{2}$ is trivial since

$$
\left(F^{2}\right)_{i k}=(R)_{i k}=\delta_{(i+k) \bmod N}, \quad 0 \leqslant i, k \leqslant N-1
$$

is the reflection operation. Finally we note that $F^{t}$ defined by (42) and (43) is a periodic matrix function with period 4 which agrees with the property $F^{4}=I$. Some corresponding identities for the coefficients of the polynomials defining various powers of $F$ include

$$
\begin{aligned}
\alpha_{l}(t+1) & =\alpha_{i}^{*}(-t), \quad \text { where } l=-(i-1) \bmod 4 . \\
\alpha_{l}(t) & =\alpha_{i}^{*}(-t), \quad \text { where } l=-i \bmod 4 .
\end{aligned}
$$

Since $F$ is unitary, $F^{t}$ is also unitary for any $t$ and Parseval's relationship remains valid between signals in complex $N$-space and their fractional DFT's computed by multiplication by $F^{t}$.

A nice geometric picture of the generalization of time and frequency domains is obtained by considering a function defined on $N$ circles of radii $0<r_{i}<r_{i+1}, 0 \leqslant i \leqslant N-1$, centered at the origin of the complex plane. The function values at the points on the positive real axis are the time domain (signal) values. The function values along a ray of angle $t \pi / 2$ with respect to the positive real axis correspond to the image of the time domain values under $F^{t}$, the fractional DFT (transform) values. Hence, every signal may be imbedded into a family of periodic functions in a way parametrized by an angle which determines a generalized frequency domain.

Several problems of signal design and analysis can now be posed. We can ask for the optimum value of $t$ (angle) for separating two signals. By this we would mean the slice of the associated function along which the fractional DFT's overlapped the least according to some measure. Cryptographic applications, where the effects of an additive "pseudonoise" sequence could be easily removed if a "key" angle $t$ were known, can be visualized. Because the computational complexity of the fractional DFT is about the same as the FFT, such methods would be practically implementable. Both of these ideas show how the concept of a generalized time and frequency domain suggests applications akin to multiplexing.

Another application for the fractional DFT is in transform coding [18], [19] for data compression purposes. Transform coding amounts to quantization of the transformed signal or data before storage or transmission. This results in lower storage or bandwidth requirements. The data may be approximately reconstructed by applying the inverse transform operation.

Various fractional DFT's could be used as part of a transform coding scheme. However, here we suggest a novel way of exploiting the fractional DFT to obtain a two-dimensional transform for this purpose. We consider the following intriguing transform pair relationships.

$$
\begin{gathered}
X_{i}=\left(\sum_{k=0}^{M-1} w^{-i k} x_{k}\right) / \sqrt{M} \\
x_{i}=\left(\sum_{k=0}^{M-1} W^{i k} X_{k}\right) / \sqrt{M} .
\end{gathered}
$$

Here $W$ is an $N \times N$ matrix which generalizes the notion of the primitive root of unity: we have $\left(I-W^{j}\right)$ invertible for $1 \leqslant j \leqslant M-1$. The "data" $\left\{x_{k}\right\}$ and "transform values" $\left\{X_{k}\right\}$ are $N$ by $L$ matrices (discrete images or subimages, for example). By taking $M>4$, an acceptable choice for $W$ is the fractional DFT $F^{t}$, where $t=4 / M$, since the eigenvalues of $F^{t}$ are [from (41)] $e^{j k \pi t / 2}, 1 \leqslant k \leqslant 4$. It is doubly intriguing to observe that the usual signal flowgraph for the FFT algorithm holds for this transform with the appropriate substitution of matrix operations with the matrix $W$ playing the role of the complex multiplications in the butterflies. From above, we know that multiplication by $W$ can, itself, be performed using FFT methods. It seems worthwhile to note that since matrix multiplication is noncommutative, there is no substantial generalization of the convolution theorem of the DFT to this matrix transform. However, for transform coding applications, only the transform pair relationship, (47), is required.

As a closing note, we might point out that other unitary transforms may be used in place of the DFT to obtain a matrix $W$ for use in the transform pair (47). The properties required are that the minimal polynomial of the transform takes the form $\lambda^{r}-1$ for small $r$ and that a fast implementation of the transform be available for computing the polynomial expression for $W$, defined as a fractional power. For example, the 
unitary Walsh-Hadamard transform [18] has $r=2$ and with $W$ equal to the $2 / M$ th power of the transform, multiplication by $W$ is performed by taking the appropriate linear combination of the signal vector and its Walsh-Hadamard transform vector.

\section{CONCLUSIONS}

We have described two applications of linear algebra and matrix theory to the DFT. Using commuting matrices, the eigenanalysis of the DFT can be performed using standard numerical methods. Fractional powers of the DFT may be easily computed; also they suggest new signal processing techniques for multiplexing and transform coding. These problems will be investigated in future research.

\section{APPENDIX I}

\section{Existence of a Matrix $\Sigma_{D}$ Having Distinct Eigenvalues}

Using perturbation methods on the matrix $S[(12)]$, we will show that it is always possible to construct a matrix $\Sigma_{D}$ as described in (7)-(11) whose eigenvalues are distinct. Let $\epsilon$ be a complex number and define the parametric family of filters $\{D(z, \epsilon)\}$ by

$$
D(z, \epsilon)=\left\{\begin{array}{c}
z+z^{-1}+\epsilon \sum_{k=-M}^{M} z^{k} \\
N \text { odd }, M=(N-1) / 2 \\
z+z^{-1}+\epsilon \sum_{k=-M}^{M} z^{k}+(\epsilon / 2)\left(z^{N / 2}+z^{-N / 2}\right) \\
N \text { even, } M=N / 2-1 .
\end{array}\right.
$$

Applying the construction of (7)-(11), we obtain the parametric family of matrices commuting with $F$

$$
\Sigma_{D}(\epsilon)=S+\epsilon S_{1}
$$

where $S$ is given by (12) and

$$
S_{1}=\left(\begin{array}{cccc}
N+1 & 1 & 1 & \\
1 & 1 & 1 & \ldots \\
1 & 1 & 1 & \\
& \vdots & & \ddots
\end{array}\right)
$$

The elements of $\Sigma_{D}(\epsilon)$ are analytic (linear!) functions of $\epsilon$, and $\Sigma_{D}(\epsilon)$ is real and symmetric for real $\epsilon$. Thus the eigenvalues and the projections onto the eigenspaces of $\Sigma_{D}(\epsilon)$ are analytic functions of $\epsilon$ in a neighborhood of 0 in the complex $\epsilon$-plane [20]. Being continuous functions of $\epsilon$, two distinct eigenvalues of $S$ cannot coalesce for small $\epsilon$, so we must show that the multiple (double) eigenvalues of $\Sigma_{D}(0)=S$ split apart for small $\epsilon$ to show that $\Sigma_{D}(\epsilon)$ has distinct eigenvalues for sufficiently small nonzero $\epsilon$.

Let $\mu$ be a double eigenvalue of $S$ with odd normalized eigenvector $u$ and even normalized eigenvector $\hat{u}$ as described in Section II. Let $\mu_{1}(\epsilon)$ and $\mu_{2}(\epsilon)$ be the analytic functions describing the eigenvalues of $\Sigma_{D}(\epsilon)$, where $\mu_{1}(0)=\mu_{2}(0)=\mu$.
Let $P(\mu, \epsilon)$ be the eigenprojection onto the space spanned by the eigenvectors of $\Sigma_{D}(\epsilon)$ corresponding to eigenvalues $\mu_{1}(\epsilon)$ and $\mu_{2}(\epsilon)$. Then [20]

$$
\left(\mu_{1}(\epsilon)+\mu_{2}(\epsilon)\right) / 2=\mu+\sum_{i=1}^{\infty} \hat{\mu}_{i} \epsilon^{i}
$$

and

$$
P(\mu, \epsilon)=P_{0}+\sum_{i=1}^{\infty} P_{i} \epsilon^{i}
$$

where

$$
P_{0}=u u^{\prime}+\hat{u} \hat{u}^{\prime}
$$

is the eigenprojection onto the eigenspace of $S$ corresponding to eigenvalue $\mu$. The coefficients of (A4) and (A5) are related by $[20,(2.1)]$

$$
\hat{\mu}_{i}=\operatorname{trace}\left(S_{1} P_{i-1}\right) / 2 i, \quad i>0 .
$$

From the odd symmetry of $u$, we have

$$
S_{1} u=0
$$

so that from (A2) $u$ is an eigenvector of $\Sigma_{D}(\epsilon)$ corresponding to eigenvalue $\mu$. Thus we take $\mu_{1}(\epsilon)=\mu$, a constant function. From (A4)

$$
\mu_{2}(\epsilon)=\mu+2 \sum_{i=1}^{\infty} \hat{\mu}_{i} \epsilon^{i}
$$

Using (A7) to evaluate $\hat{\mu}_{1}$, we find

$$
\begin{aligned}
2 \hat{\mu}_{1} & =\operatorname{trace}\left(S_{1}\left(u u^{\prime}+\hat{u} \hat{u}^{\prime}\right)\right) \\
& =\operatorname{trace}\left(S_{1} \hat{u} \hat{u}\right), \quad \text { by }(\mathrm{A} 9) \\
& =N \hat{u}_{0}^{2}+\left(\sum_{i=0}^{N-1} \hat{u}_{i}\right)^{2}
\end{aligned}
$$

where $\hat{u}=\left(\hat{u}_{0}, \hat{u}_{1}, \cdots, \hat{u}_{N-1}\right)^{\prime}$. In Section II we showed that $\hat{u}_{0} \neq 0$, so $\hat{\mu}_{1} \neq 0$ and the eigenvalue $\mu_{2}(\epsilon)$ is not a constant function. So for sufficiently small $\epsilon$, the eigenvalues $\mu_{1}(\epsilon)$ and $\mu_{2}(\epsilon)$ are distinct. This completes the argument.

\section{REFERENCES}

[1] J. H. McClellan and T. W. Parks, "Eigenvalue and eigenvector decomposition of the discrete Fourier transform," IEEE Trans. Audio Electroacoust., vol. AU-20, pp. 66-74, 1972.

[2] E. Landau, Vorlesungen über Zahlentheorie, vol. 1. New York: Chelsea, 1927/1950 (reprint), p. 164.

[3] I. J. Good, "Analogues of Poisson's summation formula," Amer. Math. Monthly, vol. 69, pp. 259-266, 1962.

[4] J. H. McClellan, "Comments on "Eigenvalue and eigenvector decomposition of the discrete Fourier transform'," IEEE Trans. Audio Electroacoust., vol. AU-21, p. 65, 1973.

[5] L. Auslander and R. Tolimieri, "Is computing with the finite Fourier transform pure or applied mathematics?" Bull. Amer. Math. Soc., vol. 1, pp. 847-897, 1979.

[6] R. Yarlagadda, "A note on the eigenvectors of DFT matrices," IEEE Trans. Acoust., Speech, Signal Processing, vol. ASSP-25, pp. 586-588, 1977.

[7] A. K. Jain. "A sinusoidal family of unitary transforms," IEEE Trans. Pattern Anal. Machine Intell., vol. PAMI-1, pp. 356-365, 1979.

[8] P. Lancaster, Theory of Matrices. New York: Academic, 1969. 
[9] J. H. Wilkinson, The Algebraic Eigenvalue Problem. Oxford, England: Clarendon, 1965.

[10] A. S. Householder, The Theory of Matrices in Numerical Analysis. New York: Blaisdell, 1964.

[11] D. Slepian and H. Landau, "A note on the eigenvalues of Hermitian matrices," SIAM J. Math. Anal., vol. 9, pp. 291-297, 1978.

[12] B. W. Dickinson and K. Steiglitz, "Eigenvalues of symmetric matrices: System theory conditions for distinctness," IEEE Trans. Automat. Contr., vol. AC-25, pp. 284-285, 1980.

[13] H. Hochstadt, "On the theory of Hill's matrices and related inverse spectral problems," Lin. Alg. Appl., vol. 11, pp. 41-52, 1975.

[14] A. Bjork and G. H. Golub, "Eigenproblems for matrices associated with periodic boundary conditions," SIAM Rev., vol. 19, pp. 5-16, 1977.

[15] A. L. Andrew, "Eigenvectors of certain matrices," Lin. Alg. Appl., vol. 7, pp. 151-162, 1973.

[16] A. Cantoni and P. Butler, "Eigenvalues and eigenvectors of symmetric centrosymmetric matrices," Lin. Alg. Appl., vol. 13, pp. $275-288,1976$.

[17] N. W. McLachlan, Theory and Application of Mathieu Functions. Oxford, England: Clarendon, 1947.

[18] N. Ahmed and K. R. Rao, Orthogonal Transforms for Digital Signal Processing. Berlin, Germany: Springer-Verlag, 1975.

[19] P. A. Wintz, "Transform picture coding," Proc. IEEE, vol. 60, pp. 809-820, 1972.

[20] T. Kato, Perturbation Theory for Linear Operators, ch. 2. New York: Springer-Verlag, 1966.

Bradley W. Dickinson (S'70-M'74) was born in St. Marys, PA, on April 28,1948 . He received the B.S. degree in engineering from Case Institute of Technology, of Case Western Reserve University, Cleveland, $\mathrm{OH}$, in 1970, and the M.S. and Ph.D. degrees in electrical engineering from Stanford University, Stanford, CA, in 1971 and 1974, respectively.

Since 1974 he has been with the Department of Electrical Engineer-

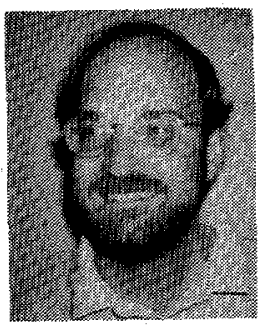

ing and Computer Science, Princeton University, Princeton, NJ, where he is now an Associate Professor.

Dr. Dickinson is a member of Tau Beta $\mathrm{Pi}$, S.A.B.R., and Sigma Xi. He has been Associate Editor for Identification for the IEEE TRANSactions on Automatic Control, and is currently a member of the Board of Governors of the IEEE Information Theory Group.

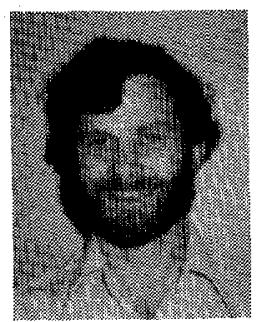

Kenneth Steiglitz (S'57-M'64-SM'79-F'81) was born in Weehawken, NJ, on January 30, 1939. He received the B.E.E. degree in 1959, the M.E.E. degree in 1960, and the Eng. Sc. D. degree in 1963, all from New York University, NY.

Since September 1963 he has been with the Department of Electrical Engineering and Computer Science, Princeton University, Princeton, NJ, where he is now Professor, teaching and conducting research in the computer and systems areas. He is Associate Editor of the Journal of the Association for Computing Machinery, and the journal Networks.

He is the author of Introduction to Discrete Systems (Wiley, 1974), and coauthor, with C. H. Papadimitriou, of Combinatorial Optimization: Algorithms and Complexity (Englewood Cliffs, NJ: Prentice-Hall, 1982).

Dr. Steiglitz is a member of the Digital Signal Processing Committee of the Acoustics, Speech, and Signal Processing Society, and has served as an Administrative Committee member and Awards Chairman of the society. He is a member of Eta Kappa Nu, Tau Beta Pi, and Sigma Xi.

\title{
Realizable Wiener Filtering in Two Dimensions
}

\author{
MICHAEL P. EKSTROM, SENIOR MEMBER, IEEE
}

\begin{abstract}
The extension of Wiener's classical mean-square estimation theory to a two-dimensional setting is presented. In analogy with the one-dimensional problem, the optimal realizable filter is derived by solution of a two-dimensional, discrete Wiener-Hopf equation using a spectral factorization procedure. Filters are developed for the cases of prediction, filtering, and smoothing, and appropriate error expressions are derived to characterize their performance.
\end{abstract}

\section{INTRODUCTION}

W HILE developed in antiquity, Wiener's minimum meansquare error (MMSE) estimation theory [1] continues

Manuscript received May 28, 1981. This work was supported in part by a Senior Scientist Award of the Alexander von Humboldt Foundation, while the author was Visiting Scientist at the Lehrstuhl für Nachrichtentechnik, Universität Erlangen-Nürnberg, Erlangen, West Germany.

The author is with Schlumberger-Doll Research, Ridgefield, CT 06877. to play a prominent role in modern time series analysis. Indeed, its extension to new applications is a topic of active research interest.

The representative problem addressed by Wiener was that of optimally estimating an unobserved time signal $s(t)$ given a noise corrupted observation, $s(t)+w(t)$, where $w(t)$ is a noise process. Both signal and noise are modeled as wide-sense stationary processes; the estimator is constrained to be linear, and derived optimal in the sense of achieving the MMSE associated with the estimate. Two classes of estimators were described by Wiener: the so-called noncausal (unrealizable, bilateral) filter which uses all past, present, and future observations in forming the estimate, and the so-called causal (realizable, unilateral) filter which uses only past and present observations.

With appropriate generalization, the fundamental estimation problem addressed by Wiener, that of estimating signals from 